Internist 2018 · 59:217

https://doi.org/10.1007/s00108-018-0389-2

Online publiziert: 20. Februar 2018

(c) Springer Medizin Verlag GmbH, ein Teil von Springer Nature 2018

CrossMark

\section{Kochanek ${ }^{1} \cdot$ M. Hallek ${ }^{1} \cdot$ H. Lehnert ${ }^{2} \cdot$ S. M. Schellong ${ }^{3}$}

${ }^{1}$ Klinik I für Innere Medizin, Universitätsklinikum Köln, Köln, Deutschland

${ }^{2}$ Medizinische Klinik I, Universitätsklinikum Schleswig-Holstein, Campus Lübeck, Lübeck, Deutschland

${ }^{3}$ Medizinische Klinik 2, Städtisches Klinikum Dresden, Campus Friedrichstadt, Dresden, Deutschland

\title{
Internistische Notfälle an der Schnittstelle von ambulant und stationär - Teil 2
}

Die Zusammenarbeit zwischen Hausarztpraxis und Krankenhaus kann Schwierigkeiten beinhalten, die auch auf fachlichen, kommunikativen oder organisatorischen Problemen basieren. Gleichwohl ist es gerade bei internistischen Notfällen wichtig, dass die Zusammenarbeit an dieser Schnittstelle funktioniert. Daher stellt die vorliegende Ausgabe von Der Internist die Frage: Was kann, soll und muss der Hausarzt in der ambulanten Versorgung tun? Was, kann, soll und muss die stationäre Versorgung leisten. Dieser zweite Teil aus der Reihe „Internistische Notfälle an der Schnittstelle von ambulant und stationär" (erster Teil erschien in Ausgabe 9, September 2017) befasst sich mit den Themenkomplexen Fieber, auffälliges Bein und auffällige Laborwerte. Wie auch schon in Teil I wurden Symptome und nicht Krankheitsbilder gewählt, denn diese spiegeln sicher mehr den realistischen Alltag an dieser Schnittstelle wider.

Die Herangehensweise des ambulant tätigen Arztes bezüglich der gewählten Themenkomplexe wird mit dem Vorgehen des stationär arbeitenden Arztes verglichen. Ein besonderes Ziel der Autoren ist, die Symptome zu besprechen und ein entsprechendes „work-up“ der dazugehörigen Erkrankungen aufzuzeigen. Der Arbeitsplan ist genau auf die Schnittstelle von ambulant und stationär zugeschnitten.

Warum ist dies so wichtig? Einerseits geht es um eine optimale Versorgung des Patienten: schnell und möglichst effektiv, also gleichzeitig fehlerfrei. Andererseits sollen Kosten und wertvolle personelle
Ressourcen der Arztpraxen, hausärztlichen Notfallpraxen, Notfallambulanzen in den Krankenhäusern oder auch der Intensivstationen nicht unnötig durch eine inadäquate Diagnostik und Therapie belastet werden. Besonders deutlich wird dies an der Schnittstelle zentrale Notaufnahme. Neuere Auswertungen zeigen, dass sich pro Jahr mit steigender Tendenz etwa 17-20 Mio. Patienten dort vorstellen $[1,2]$. Die Gründe sind vielfältig. Durch eine bessere Verzahnung der ambulanten und stationären Versorgung ließen sich sicherlich viele dieser Patienten effizienter versorgen. Die zunehmende Belastung der Notaufnahmen würde reduziert. Die Beiträge in dieser Ausgabe werden sicherlich die strukturellen Probleme nicht lösen können. Sie sollen aber dazu beitragen, dass typische Probleme unserer Patienten im klinischen Kontext optimal angegangen werden.

Bei der Diskussion und Vorbereitung der beiden Ausgaben von Der Internist ist uns deutlich geworden, wie wichtig das Thema der internistischen Notfälle an der Schnittstelle von ambulant und stationär ist. Wir wünschen Ihnen beim Lesen viel Spaß und wertvolle Erkenntnisse, auch in der Hoffnung, damit einen fruchtbaren Austausch zwischen ambulanter und stationärer Versorgung befördern zu können.

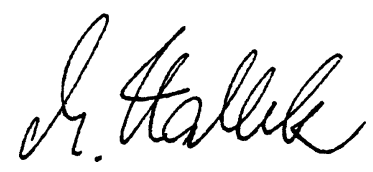

M. Hallek

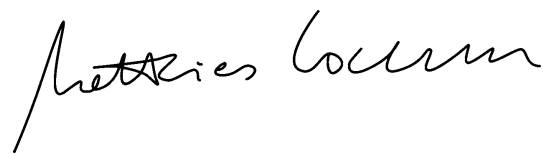

M. Kochanek

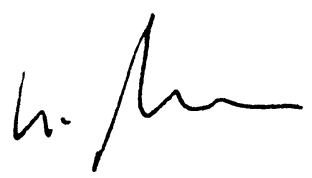

H. Lehnert

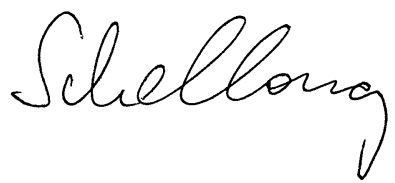

S.M. Schellong

\section{Korrespondenzadresse}

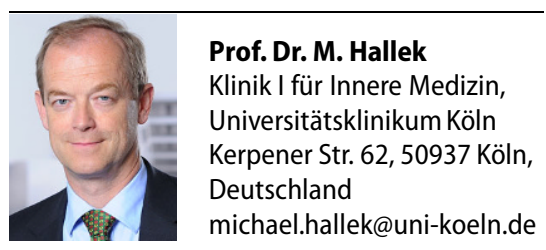

Interessenkonflikt. M. Kochanek, M. Hallek, H. Lehnert und S.M. Schellong geben an, dass kein Interessenkonflikt besteht.

\section{Literatur}

1. Zi Report der Kassenärztlichen Vereinigung ISSN 2199-1480 (online). https://www.zi.de/ $\mathrm{cms} /$ fileadmin/images/content/Publikationen/ Zi-Paper_11-2017_Notfallversorgung.pdf.Zugegriffen: $1 \overline{6} .01 .18$

2. Pressemitteilung DGINA. https://www.dgina.de/ news/geringe-auslastung-von-notaufnahmendgina-widerspricht-zi-paper_62. Zugegriffen: 16.01.18 